\title{
Legal and Ethical Challenges in Multimedia Research
}

\author{
Vivek K. Singh \\ School of Comm. \& Information \\ Rutgers University \\ New Brunswick, NJ, USA \\ v.singh@rutgers.edu \\ Mireille Hildebrandt \\ Vrije Universiteit Brussel. \\ Belgium, and Radboud University, \\ the Netherlands \\ m.hildebrandt@cs.ru.nl
}

\author{
Elisabeth André \\ Faculty of Applied Informatics \\ University of Augsburg \\ Augsburg, Germany \\ andre@informatik.uni- \\ augsburg.de \\ David A. Shamma \\ FX Palo Alto Laboratory (FXPAL) \\ Palo Alto, CA, USA \\ aymans@acm.org
}

\author{
Susanne Boll \\ Department of Computer Science \\ University of Oldenburg \\ Oldenburg, Germany \\ Susanne.Boll@uol.de
}

\author{
Tat-Seng Chua \\ Department of Computer Science \\ National University of Singapore \\ Singapore \\ chuats@comp.nus.edu.sg
}

\begin{abstract}
Multimedia research has now moved beyond laboratory experiments and is rapidly being deployed in real-life applications including advertisements, social interaction, search, security, automated driving, and healthcare. Hence, the developed algorithms now have a direct impact on the individuals using the abovementioned services and the society as a whole. While there is a huge potential to benefit the society using such technologies, there is also an urgent need to identify the checks and balances to ensure that the impact of such technologies is ethical and positive. This panel will bring together an array of experts who have experience collecting large-scale datasets, building multimedia algorithms, and deploying them in practical applications, as well as, a lawyer whose eyes have been on the fundamental rights at stake. They will lead a discussion on the ethics and lawfulness of dataset creation, licensing, privacy of individuals represented in the datasets, algorithmic transparency, algorithmic bias, explainability, and the implications of application deployment. Through an interactive process engaging the audience, the panel hopes to: increase the awareness of such concepts in the multimedia research community; initiate a discussion on community guidelines all for setting the future direction of conducting multimedia research in a lawful and ethical manner.
\end{abstract}

\section{CCS CONCEPTS}

- $\mathrm{CCS} \rightarrow$ Social and professional topics $\rightarrow$ Computing / technology policy

Permission to make digital or hard copies of part or all of this work for personal or classroom use is granted without fee provided that copies are not made or distributed for profit or commercial advantage and that copies bear this notice and the full citation on the first page. Copyrights for third-party components of this work must be honored. For all other uses, contact the Owner/Author.

$M M$ '19, October 21-25, 2019, Nice, France

(c) 2019 Copyright is held by the owner/author(s).

ACM ISBN 978-1-4503-6889-6/19/10.

https://doi.org/10.1145/3343031.3351493
- CCS $\rightarrow$ Security and privacy $\rightarrow$ Human and societal aspects of security and privacy

\section{KEYWORDS}

Ethics; law; multimedia; fairness; accountability; transparency

\section{ACM Reference format:}

Vivek K. Singh, Elisabeth André, Susanne Boll, Mireille Hildebrandt, David A. Shamma, and Tat-Seng Chua. 2019. Panel: Legal and Ethical Challenges in Multimedia Research In Proceedings of ACM Multimedia conference (MM'19), Oct 21-25, 2019, Nice, France. ACM, New York, NY, USA, 2 pages. https://doi.org/10.1145/3343031.3351493

\section{INTRODUCTION}

Recent growth spurt in multimedia research has led to some exciting developments in terms of multimedia search, selfdriving cars, and medical analysis. At the same time, there have been recent reports questioning both the processes and the outcomes for the developed technologies. For instance, [1] questions the ethics of using public image data in YFCC100M and IBM's Diversity in Faces datasets for training face recognition algorithms. Similar concerns have been raised about the outcomes of the developed algorithms. For instance, [2] has reported that face detection algorithms work much more accurately for white men than dark-skinned women. Further, multiple authors have criticized the use of video analysis software for automatic tracking of people in both civilian and military settings [3]. In fact, San Francisco in the US Silicon Valley has banned the use of face recognition technology for government applications [4]. Meanwhile the Data Protection Authority in Hamburg, Germany, has ordered Google to ban its employees 'from listening to and reviewing EU data subjects' voice recordings for 3 months, to investigate potentially unlawful processing under the GDPR [5].

Each of these issues raises an important ethical concern, while obviously nobody is above the law, so keen attention must be paid to legal demands and obligations. Many times the 
opinions of the experts in the multimedia community might not match with those in the popular media, noting that many of these concerns might already have legal guidelines. There is an important need for the multimedia research community as a whole to have free and frank discussions on this topic and lead the charge in educating the rest of the world on the potential benefits and harms of such technologies rather than reacting to the news stories. Equally importantly, the multimedia research community needs to identify a set of community norms and guidelines on the processes and the outcomes of the technologies being developed.

\section{PANEL}

This panel will include the following participants.

\section{Vivek K. Singh, Rutgers University}

Vivek Singh is an Assistant Professor in the School of Communication and Information at Rutgers University. His work on privacy and computational social science in large-scale data analysis has been published in leading scientific venues (e.g., Science, Proc. of the IEEE), and was cited by the US appellate court in its decision on the surveillance of US citizen's phone data. He will moderate the discussion.

\section{Elisabeth André, Augsburg University}

Elisabeth André is a Professor of Computer Science and the Chair of Human-Centered Multimedia in the Faculty of Applied Informatics at Augsburg University, Germany. Her research interests include multimodal user interfaces, affective computing, and social signal processing. In 2019, she was named one of the ten most influential figures in the history of $\mathrm{AI}$ in Germany by the National Society for Informatics (GI). She is the Editor-in- Chief of IEEE Transactions on Affective Computing.

\section{Susanne Boll, University of Oldenburg}

Susanne Boll-Westermann is a Professor for Multimedia and Internet in the Department of Computing Science at the University of Oldenburg, Germany. Her main research interests are semantic information retrieval and human computer interaction. Recently, she worked on the social acceptability of body-worn cameras. She contributed to about 200 scientific papers and organized a number of conferences and workshops (including serving as a co-chair for ACM Multimedia 2018).

\section{Tat-Seng Chua, National University of Singapore}

Tat-Seng Chua is the KITHCT Chair Professor at the School of Computing, National University of Singapore. His main research interest is in multimedia information retrieval and social media analytics. In particular, his research focuses on the extraction, retrieval and question-answering of text, video and live media arising from the Web and social networks. He was the recipient of ACM SIGMM Technical Achievement Award 2015.

\section{Mireille Hildebrandt, Vrije Universiteit Brussel, Radboud University}

Mireille Hildebrandt is a Research Professor on 'Interfacing Law and Technology' at the Faculty of Law and Criminology of Vrije Universiteit Brussel (VUB), appointed by the VUB Research Council and part-time full professor of law at the Department of Computer Science at Radboud University. Her research interests concern the implications of automated decisions, machine learning and mindless artificial agency for law and the rule of law in constitutional democracies. In 2018 she received an ERC Advanced Grant for her project on 'Counting as a Human Being in the era of Computational Law' (2019-2024): www.cohubicol.com.

\section{David A. Shamma, FXPAL}

David A. Shamma is a senior research scientist at FX Palo Alto Laboratory (FXPAL) and a distinguished member of the ACM. He investigates social computing systems (how people interact, engage, and share media experiences both online and in-the-world) through three avenues: AI, systems \& prototypes, and qualitative research. He led the creation of the YFCC100M image and video dataset and has served as a Program Co-Chair for ACM Multimedia Conference 2013.

\section{REFERENCES}

[1] If your image is online, it might be training facial-recognition AI https://www.cnn.com/2019/04/19/tech/ai-facial-recognition/index.html

[2] Buolamwini, J., \& Gebru, T. (2018, January). Gender shades: Intersectional accuracy disparities in commercial gender classification. In Conference on Fairness, Accountability and Transparency (pp. 77-91).

[3] Facial recognition poses serious risks. Congress should do something about it. https://www.washingtonpost.com/opinions/facial-recognition-posesserious-risks-congress-should-do-something-about-it/2018/07/18/c4c8973c89c1-11e8-a345-a1bf7847b375_story.html?utm_term=.8a991491dd14

[4] San Francisco just banned facial-recognition technology. https://www.cnn.com/2019/05/14/tech/san-francisco-facial-recognitionban/index.html

[5] https://globaldatareview.com/article/1195881/hamburg-regulator-bansgoogle-from-listening-to-smart-speaker-audio. 